\title{
L’impact économique du développement des marques de distributeurs
}

Fabian Bergès-Sennou, Philippe Bontems et Vincent Réquillart

Université de Toulouse (GREMAQ-UMR INRA 1291, IDEI)

Avril 2007

Adresse postale :

Vincent REQUILLART

Université de Toulouse (GREMAQ, IDEI)

UMR INRA 1291

Manufacture des Tabacs. Aile Jean-Jacques Laffont

21 Allée de Brienne

31000 Toulouse, France

Tel : 0561128607

FAX : 0561128637

Mail : Vincent.Requillart@toulouse.inra.fr

Résumé

Cet article constitue une synthèse de la littérature traitant des marques de distributeurs (MDD). Dans une première partie, nous faisons l'état des lieux statistiques sur l'importance des MDD. Dans un second temps, les raisons ayant poussé les distributeurs à introduire les MDD sont analysées sur un plan théorique et leurs conclusions confrontées à la littérature empirique sur les MDD. L'accent est mis plus particulièrement sur les relations verticales et le choix des caractéristiques des produits. L'impact du développement des MDD sur les firmes amont est également abordé. Enfin, une réflexion sur les effets de bien-être du développement des MDD est amorcée.

Mots clés : économie industrielle, relations verticales, marques de distributeurs, agro-alimentaire.

JEL : L22, L66, Q18

\section{Summary}

This article is a survey of the economic literature on private labels (PL). In the first section, we look at statistics on private label development. Then, the causes of private label introduction by retailers are analyzed on a theoretical ground, and conclusions are confronted with empirical studies findings. We focus on vertical relationships and product characteristics choices. Last, we give some thoughts on PL and their impact on social welfare.

Keywords: industrial organization, vertical relationships, private labels, agrofood. 
L’impact économique du développement des marques de distributeurs

L'issue de cette lutte ne semble ni prochaine ni même vraisemblable. Ce qu'il faut retenir, c'est que marque et publicité ont mis entre les mains de l'industrie un atout maître qui lui a permis de se relever de son ancienne infériorité vis-à-vis du commerce et de se mesurer à armes égales avec lui. Elles lui auraient même donné la suprématie si la naissance du grand commerce de détail n'était venue se mettre en travers, en opposant un contrepoids sérieux à la puissance nouvellement conquise du fabricant. Certains sont sûrs que l'industrie, de plus en plus concentrée, parviendra un jour à régir à sa guise l'ensemble du commerce grâce à la publicité. C'est là, croyons-nous, méconnaître singulièrement la puissance et les moyens d'actions du grand commerce de détail. Il ne peut être question d'une victoire décisive d'une des deux parties. Continuellement se poursuivront attaques et contre-attaques. Ici c'est la marque du fabricant qui s'imposera au commerce ; là c'est la marque du commerce qui l'emportera. La suprématie de l'un des deux adversaires ne peut être que partielle et d'importance variable suivant les branches et les régions. Le tout dépendra surtout de la valeur des méthodes de vente employées par chacun. (Francis Elvinger, 1928, « La marque » texte tiré de Baroux, 05 / 01 / 2000, Les échos, Deux siècles de bras de fer entre l’industrie et le commerce).

\section{Introduction}

La Loi du 15 mai 2001 relative aux Nouvelles Régulations Economiques (NRE) définit la marque de distributeur (MDD) dans son article 62 :

«Est considéré comme produit vendu sous marque de distributeur le produit dont les caractéristiques ont été définies par l'entreprise ou le groupe d'entreprises qui en assure la vente au détail et qui est le propriétaire de la marque sous laquelle il est vendu ».

Selon l'entreprise Nielsen, les MDD sont des marques appartenant à une entreprise commerciale de vente au détail pour une ligne de produits distribuée exclusivement par cette dernière ou sous son contrôle. PLMA (Private Label Manufacturers Association) en donne une définition voisine: les produits MDD comprennent l'ensemble des marchandises vendues sous la marque d'un distributeur. ${ }^{1}$ Cette marque peut être le nom du distributeur lui-même ou une appellation créée exclusivement à l'usage de ce dernier. ${ }^{2}$

Ces définitions mettent en avant deux points essentiels : d'une part, le contrôle de la marque par le distributeur alors que ce contrôle est traditionnellement du ressort d'un producteur et d'autre part, la notion d'exclusivité qui implique que les différents distributeurs ne fourniront pas les mêmes MDD, ce qui n'est pas le cas lorsque les distributeurs distribuent des marques de producteurs, souvent 
dénommées marques nationales $(\mathrm{MN})$ dès qu'elles sont largement diffusées sur le territoire. Ainsi les MDD sont susceptibles de modifier non seulement les relations entre distributeurs et producteurs puisque le distributeur étend son rôle, mais aussi les relations entre distributeurs en introduisant un élément supplémentaire de différenciation des distributeurs.

La modification des relations verticales entre producteurs et distributeurs s'exerce via la concurrence en aval que se livrent les marques de distributeurs et les marques nationales qui sont des produits substituables. En possédant leur propre marque, les distributeurs ont ainsi une alternative à la marque nationale. La négociation avec l'amont s'en trouve évidemment affectée. Les MDD jouent également un rôle dans la concurrence entre distributeurs car elles permettent à ces derniers d'élargir leur gamme avec des produits qui ne sont pas directement comparables entre les détaillants. ${ }^{3}$ Pour les consommateurs, les MDD représentent potentiellement un bien supplémentaire susceptible d'accroître leur surplus à travers l'effet variété. Pour les firmes amont productrices de MN, le développement de ces produits concurrents leurs sont a priori préjudiciables mais dans une moindre mesure si elles produisent également les marques propres des distributeurs.

Une littérature traitant de ces questions s'est développée ces dix dernières années. En effet, les résultats de la littérature sur les restrictions verticales ne sont pas directement transposables à l'analyse économique des MDD dans la mesure où, comme le souligne Rey (1997), la marque est généralement assimilée au producteur amont. Une des caractéristiques fondamentales des MDD : le distributeur est dépositaire de la marque, n’est pas donc pas intégrée dans ces travaux. La MDD a un rôle pour le distributeur qui va bien au-delà de la simple commercialisation d’un bien supplémentaire.

Cet article fait le point sur la littérature économique prenant en compte les spécificités des MDD, il fait suite et actualise un premier article (Bergès-Sennou et al. 2004) consacré à la même question en apportant de nombreuses références publiées depuis cette date. Compte tenu des apports récents de la littérature, le plan d'analyse confronte les résultats des études théoriques aux études empiriques les plus récentes. La première section de cet article fait l'état des lieux statistiques sur l'importance du développement des MDD. Ensuite, nous analysons en détail les raisons ayant poussé les distributeurs à introduire des MDD. L'accent est plus particulièrement mis sur les relations verticales et le choix des caractéristiques des produits. L'arbitrage entre gain en pouvoir de négociation et discrimination de la demande est notamment mis en avant. Dans la mesure du possible, nous confrontons les prédictions théoriques aux résultats d'études empiriques. Une troisième partie traite des enjeux de la production des MDD pour les producteurs en amont. Enfin, nous concluons par une réflexion sur l'impact des MDD sur le surplus social et proposons quelques pistes de recherche.

\section{Faits et chiffres sur l'importance du développement des MDD}

En Europe, l'importance du développement des MDD est variable selon les pays (Tableau 1). On notera que les parts de marché en volume sont supérieures aux parts de marché en valeur signifiant 
que ces produits sont vendus en moyenne à un prix inférieur à la moyenne du prix de vente de l'ensemble des produits. La comparaison entre pays européens n'est pas toujours aisée car la définition exacte peut varier d'un pays à l'autre mais aussi d'un institut à l'autre (cf. les différences d'estimation en France entre Nielsen et Secodip). La principale différence concerne la prise en compte ou non des produits dits premiers prix ${ }^{5}$. Dans le tableau qui suit, les premiers prix sont inclus dans les part de marché des MDD.

Tableau 1 : Taux de pénétration des MDD en 2004 dans quelques pays européens (\%)

\begin{tabular}{|l|c|c|c|}
\hline \multicolumn{1}{|c|}{ Pays } & Volume & Valeur & Vol / Val \\
\hline Allemagne & 38,3 & 29,6 & 129 \\
\hline Belgique & 40,9 & 29,3 & 140 \\
\hline Espagne & 31,6 & 22,7 & 139 \\
\hline France & 30,8 & 23,5 & 131 \\
\hline Italie & 15,6 & 13,4 & 116 \\
\hline Pays-Bas & 22,5 & 21,2 & 106 \\
\hline Royaume-Uni & 41,5 & 38,0 & 109 \\
\hline
\end{tabular}

Source : PLMA / AC Nielsen 2005.

Figure 1: Part de marché des MDD et concentration du secteur de la distribution en 2004

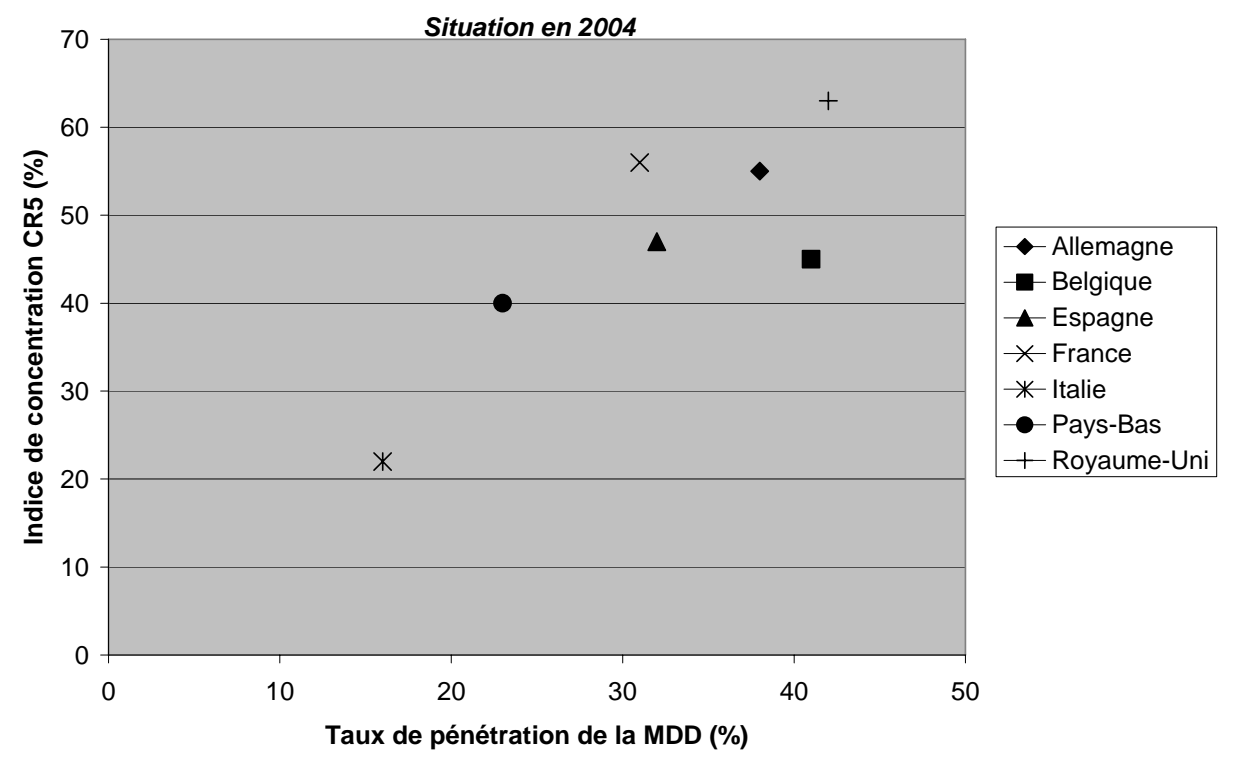

Source : Office of Fair Trading 2006 (p. 14) et PLMA 2005.

De façon générale, plus le secteur de la distribution est concentrée plus les MDD ont une place importante (Figure 1). A notre connaissance, peu de travaux ont cherché à expliquer cette corrélation. McGoldrick (1984) indique que plus le secteur est concentré, plus l'enseigne pourra aisément 
capitaliser son image sur les produits de sa gamme la dispersion dans l'esprit des consommateurs étant moindre.

Au sein d'un pays, l'importance des MDD est très variable selon les catégories de produits et au sein des catégories selon les produits. De très nombreux facteurs peuvent jouer pour expliquer une plus ou moins grande pénétration des MDD. Certains arguments renvoient à l'offre (structure de l'offre, capacité d'entrer sur le marché, politique d'innovation, etc) et d'autres aux caractéristiques de la demande. Par exemple, les biens pour lesquels les attributs confiance semblent importants (produits de beauté, alimentation infantile) enregistrent des taux de pénétration des MDD faibles (Tableaux 2$)^{6}$. Au cours des années récentes, les parts de marché des MDD sont en augmentation et ceci pour la majorité des produits.

Tableau 2 : Evolution des parts de marché en valeur des MDD par rayon en France

\begin{tabular}{|l|c|c|c|c|}
\hline \multicolumn{1}{|c|}{ Rayon } & $\mathbf{1 9 9 7}$ & $\mathbf{2 0 0 0}$ & $\mathbf{2 0 0 4}$ & $\mathbf{2 0 0 4 / 1 9 9 7}$ \\
\hline Epicerie & 18,7 & 21,8 & 24,4 & 130 \\
\hline Liquides & 18,6 & 19,9 & 21,3 & 115 \\
\hline Entretien & 20,0 & 24,8 & 28,5 & 143 \\
\hline Hygiène - Beauté & 5,9 & 7,3 & 9,3 & 158 \\
\hline Surgelés & 29,0 & 33,8 & 41,0 & 141 \\
\hline Saurisserie & 28,6 & 32,9 & 43,5 & 152 \\
\hline Crémerie & 24,2 & 26,4 & 30,4 & 126 \\
\hline Fromage Libre Service & 18,8 & 21,5 & 30,0 & 160 \\
\hline Charcuterie Libre Service & 24,2 & 37,7 & 45,9 & 190 \\
\hline Traiteur Libre Service & 17,2 & 26,4 & 32,6 & 190 \\
\hline
\end{tabular}

Source : Linéaires d'après SECODIP

De même les différentes enseignes ont des stratégies diverses vis-à-vis des marques de distributeurs. Ceci se traduit par un développement hétérogène des MDD dans les enseignes (Tableau 3). Historiquement, certaines enseignes (Carrefour, Intermarché notamment) ont introduit les MDD plus tôt (Leclerc et Auchan les ont introduit plus tardivement). De même, la politique de dénomination des MDD n'est pas identique chez tous les détaillants. Cette politique peut varier dans le temps comme le montre l'exemple d'Auchan qui a réorganisé dans les années 2000 sa gamme de MDD en apposant le nom de l'enseigne alors qu'il avait opté jusqu'à cette date pour des marques déclinées par gamme à l’instar d'Intermarché. 
Tableau 3 : Parts de Marché des MDD par enseigne en 2003, données par groupes

\begin{tabular}{|l|c|}
\hline \multicolumn{1}{|c|}{ Enseigne } & \multicolumn{1}{c|}{$\begin{array}{c}\text { Part de marché } \\
\text { MDD en volume }\end{array}$} \\
\hline Carrefour & $24,1 \%$ \\
\hline Champion & $21,4 \%$ \\
\hline Système U & $23,1 \%$ \\
\hline Leclerc & $25,4 \%$ \\
\hline Géant et Casino & $19,8 \%$ \\
\hline Monoprix / Prisunic & $15,4 \%$ \\
\hline Match & $17,4 \%$ \\
\hline Cora & $19,0 \%$ \\
\hline \hline Auchan & $23,9 \%$ \\
\hline Intermarché & $32,6 \%$ \\
\hline
\end{tabular}

Source : Sécodip / Origine : distributeurs

\section{Pourquoi les distributeurs développent des MDD?}

Selon une enquête LSA/Fournier (1996), les principales motivations des distributeurs à l'égard des MDD étaient : proposer des prix bas (33\%), améliorer les marges (25\%), renforcer l'image (18\%), fidéliser la clientèle (16\%). Ces motivations renvoient à deux types d'arguments économiques qui sont analysés en détail dans cette section. Le premier argument concerne les relations verticales entre producteurs et distributeurs. En effet, de la concurrence entre MN et MDD naît un changement du rapport de force à l'avantage du distributeur. Le second argument concerne la concurrence entre distributeurs. Chaque MDD est spécifique d'un distributeur et permet de relâcher la concurrence entre enseignes.

Ces deux éléments sont reliés car le niveau de concurrence en aval a des répercussions importantes sur le partage des profits au sein des filières verticales. Ainsi pour Steiner (1985), le pouvoir relatif entre producteurs et distributeurs est directement lié à l'attachement des consommateurs aux produits ou aux magasins:

"If consumers are more disposed to switch brands within store than stores within brand, retailers dominate manufacturers. Retails margins will be relatively high and those of manufactures relatively low. When consumers are more disposed 
to switch stores within brand than brands within store, the above market power and margin are reversed »(Steiner, 1985, p. 157-158).

Le développement d'une MDD en incitant les consommateurs à ne pas changer de magasin pour trouver ailleurs leur marque 'préférée' est donc susceptible de renforcer le pouvoir de négociation du distributeur. ${ }^{7}$ Le choix des caractéristiques de la MDD est donc un élément particulièrement stratégique pour le distributeur.

\subsection{Les MDD comme armes de négociation avec les producteurs amont}

L’idée centrale est que le distributeur, en introduisant une MDD, devient concurrent de son fournisseur. La conséquence en est que le détaillant renforce son pouvoir de négociation dans sa relation avec le producteur au sein d'une structure verticale. Schématiquement, les MDD vont être un outil aux mains des distributeurs pour d'une part discriminer la demande (en proposant un nouveau bien) et pour d'autre part améliorer en leur faveur le partage des profits au sein d'une structure verticale. $^{8}$ Nous verrons que ces deux forces sont antagonistes et que le choix optimal du positionnement de la MDD résultera d’un compromis entre ces deux objectifs.

Nous présentons tout d'abord les articles théoriques qui vont permettre de tirer des conclusions sur les profits des acteurs et les prix suite au développement des MDD. Ensuite, nous confronterons ces prédictions aux travaux empiriques concernant les MDD.

\subsubsection{Les effets de l'introduction d'une MDD dans le cadre d'une tarification linéaire}

Le cadre théorique généralement utilisé est celui d’une structure verticale composée d'un producteur et d'un distributeur tous deux en position de monopole. Le producteur produit un bien de qualité haute donnée à coût marginal constant. Il vend ce bien au distributeur à un certain prix de gros (on suppose dans un premier temps une tarification linéaire). Le distributeur, qui pour simplifier l'analyse ne supporte pas de coût de transformation, revend ce bien aux consommateurs à un certain prix de détail. ${ }^{9}$ Dans ce cadre, le prix au consommateur résulte d'une double marginalisation. En effet, chaque acteur tarifie au dessus du coût marginal pour tirer parti de son pouvoir de marché. Cette situation est défavorable pour les consommateurs mais aussi pour la structure verticale en raison de la mauvaise coordination entre producteur et distributeur. Si le distributeur introduit un nouveau bien, substitut du bien existant, on peut assister alors à une augmentation des profits du distributeur au détriment du producteur amont et à une réduction de la double marginalisation. En effet, la concurrence exercée par le nouveau bien limite le pouvoir de marché du producteur amont qui réduit 
alors son prix de gros ce qui réduit le phénomène de double marginalisation. Sauf à considérer un coût d'introduction de la MDD trop important, le surplus des consommateurs augmente du fait de la diminution de la double marginalisation et d'une plus grande variété des produits disponibles.

Dans le cadre de la littérature sur les MDD, le bien substitut est supposé être de qualité inférieure au bien initial. Cela est souvent conforme aux faits dans la mesure où les consommateurs perçoivent généralement les MDD comme étant de qualité moindre, ou tout au moins pas supérieure, aux marques nationales $(\mathrm{MN}){ }^{10}$

La littérature considère également que la MDD est achetée par le distributeur au coût marginal de production ce qui représente soit le cas où celle-ci est produite par une frange concurrentielle de petites firmes (ce qui est assez fréquent dans l'agroalimentaire dans la mesure où, sauf exception, il y a relativement peu de barrières à l'entrée), soit le cas où la firme est intégrée au distributeur et où le prix de cession interne est le coût marginal.

Dans un tel cadre, le producteur amont propose un prix de gros pour la MN. Le distributeur a ensuite le choix de référencer ou non la MN et de distribuer ou non la MDD. Enfin le distributeur fixe le prix de vente du (des) bien(s) au consommateur. Trois possibilités s’offrent alors au producteur de $\mathrm{MN}$ :

- $\quad$ proposer un prix de monopole identique à celui en vigueur lorsqu'il n’y a pas de MDD ;

- proposer un prix de gros suffisamment bas qui dissuade le distributeur d'introduire effectivement la MDD ;

- proposer un prix de gros qui tient compte de l'introduction de la MDD (il accommode l'entrée).

Deux hypothèses polaires concernant les coûts de production de la MDD ont été étudiées dans la littérature. Elles ne conduisent pas exactement aux mêmes conclusions.

Cas 1 : Le coût de la qualité est associé à des coûts fixes

Mills (1995) analyse le cas où les coûts de production des deux biens diffèrent uniquement par les coûts fixes. ${ }^{12}$ A l'inverse, les coûts variables de production de la MN et de la MDD sont identiques. Dans ce cadre d’hypothèses, Mills montre :

- Si la qualité de la MDD est faible (par rapport à la qualité de la MN), le distributeur n'introduit pas la MDD car celle-ci est un substitut de trop basse qualité par rapport à la MN. Le producteur de MN demeure donc en situation de monopole et fixe le prix de gros au niveau du prix de monopole.

- Au delà d'un certain seuil de qualité pour la MDD, le distributeur faisant face au prix de monopole pour la MN aurait intérêt à introduire la MDD. Le producteur de MN est alors incité à baisser son prix pour empêcher le distributeur d'introduire la MDD. Il pratique donc un prix limite qui 
dissuade le distributeur d'introduire effectivement la MDD. Par contre, cette menace a pour effet d'entraîner une diminution du prix de gros de la MN. Cette diminution est d'autant plus forte que la qualité de la MDD est forte (relativement à celle de la MN).

- Enfin, au delà d'un second seuil de qualité, la qualité de la MDD est trop forte pour que le producteur de MN ait intérêt à empêcher l'entrée de la MDD. Il est préférable pour lui de pratiquer un prix de gros qui ‘accommode’ l'entrée. Les deux produits sont alors effectivement présents sur le marché. Enfin, plus la qualité de la MDD augmente plus le prix de gros de la MN diminue. Lorsque les qualités perçues sont équivalentes, le prix de gros de la MN est égal à son coût marginal et le producteur amont réalise un profit nul. ${ }^{13}$

Ainsi, la menace d'introduction ou l'introduction effective de la MDD conduisent à une baisse du prix de gros d'autant plus forte que la qualité de la MDD est élevée. Les profits de la structure verticale s'accroissent (en raison de la diminution de la double marginalisation), le profit du distributeur augmentant plus que la perte du producteur. ${ }^{14}$ Les consommateurs bénéficient également de l'introduction (ou de la menace d'introduction) de la MDD : le prix à la consommation de la MN diminue et la couverture du marché s’accroît.

La prise en compte des coûts fixes ne permet pas de conclure systématiquement sur l'intérêt de l'introduction des MDD en terme de surplus social. L'effet variété des produits et baisse de la double marginalisation s'opposent aux coûts d'introduction de la MDD. Cela étant, tant que les coûts fixes sont faibles, les MDD améliorent le surplus social.

Cas 2 : Le coût de la qualité est associé à des coûts variables

Avec des coûts marginaux indépendants de la qualité, une MDD de basse qualité ne peut être compétitive vis-à-vis d'une MN (Mills, 1995). Toutefois, produire un bien de qualité inférieure peut, en pratique, permettre de limiter les coûts (par ex. en utilisant des matières premières de moindre qualité et donc moins coûteuses). Bontems, Monier et Réquillart (1999) examinent précisément une situation où le coût marginal (constant) de production d'un bien augmente avec son niveau de qualité.

De plus, il n' y a pas de raison a priori de considérer qu'à qualité identique, MN et MDD puissent être produites dans des situations de coûts similaires. Une situation possible est celle où la MDD a un désavantage en coût : à qualité identique, il est plus coûteux de produire la MDD que la MN. Cela peut s'expliquer par l'existence de gains d'apprentissage que l'entrée tardive de la MDD ne permet pas de capitaliser. Dans ce cas,

- Si la qualité de la MDD est faible, la MN ne peut empêcher l'entrée de ce concurrent à bas coût. Il est même possible que la MN soit évincée du marché lorsque le coût de la MDD est faible et que les consommateurs valorisent peu la qualité. Quand le distributeur vend les deux produits, le prix 
de la MN est décroissant avec la qualité de la MDD en raison de la concurrence plus forte exercée par celle-ci. ${ }^{15}$

- Pour des valeurs intermédiaires de la qualité de la MDD, on retrouve une stratégie de prix limite de la part du producteur de MN, comme c'était le cas précédemment. La MDD n’est donc pas introduite par le distributeur. Toutefois, la dissuasion de l'entrée de la MDD est pour le producteur de MN de moins en moins coûteuse. C’est la conséquence directe de la croissance du coût marginal de production de la MDD avec la qualité. A partir d'un certain seuil de qualité de la MDD, la MN retrouve alors son monopole naturel.

Une seconde situation est celle où MN et MDD ont des coûts de production identiques (à qualité égale). ${ }^{16}$ C’est le cas par exemple lorsque les technologies utilisées sont génériques. Dans ce cas, la MDD est toujours introduite et le prix de la MN est décroissant avec la qualité de la MDD. ${ }^{17}$

L’introduction de la MDD (ou la menace d'introduction) améliore le surplus social, car il évite ou atténue le phénomène de double marginalisation. L'absence d'introduction d'une MDD dans certains secteurs ne signifie donc pas que le distributeur soit démuni de ce levier pour augmenter son pouvoir de négociation.

Ce modèle qui repose également sur l'hypothèse de tarifs linéaires entre un producteur et un distributeur permet de mettre en évidence le rôle important joué par la structure de coûts de production sur les implications de l'introduction des MDD. Il conclut néanmoins de façon générale à l'intérêt du développement des MDD en terme de bien-être. Cette conclusion, comme dans le modèle précédent, est une conséquence directe de la diminution du phénomène de double marginalisation. La Figure 2 résume les équilibres en fonction de la qualité de la MDD ( $\mathrm{q}_{\mathrm{MDD}}$ ) pour les deux articles présentés (les seuils d'introduction ne sont toutefois pas comparables entre les deux modèles).

Figure 2: Equilibres suivant l'hypothèse de coût sur la qualité.

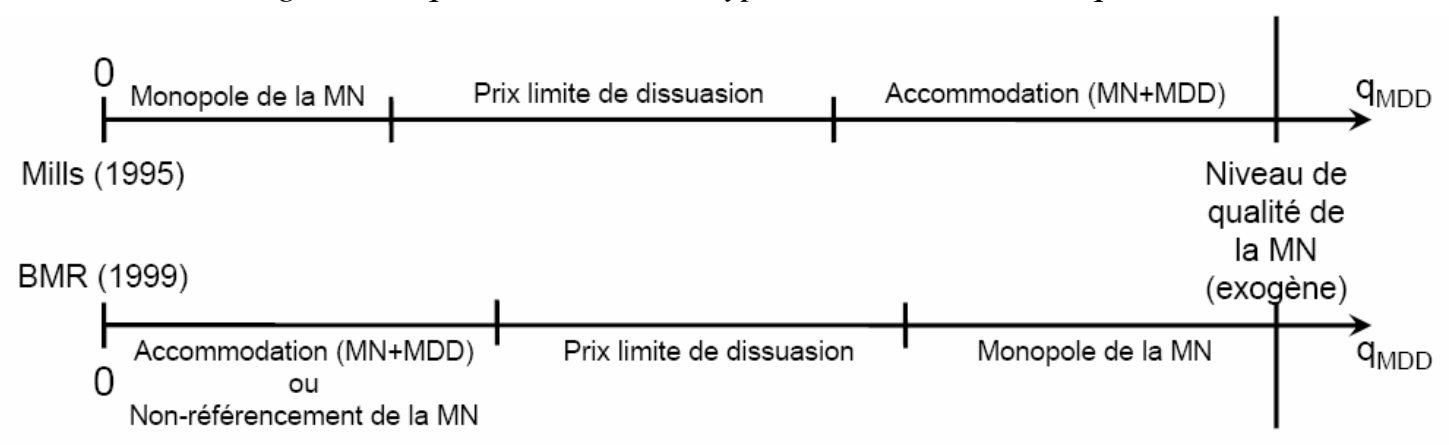

Dans les deux modèles, qu'il s’agisse de Mills (1995) ou BMR (1999), la structure de coût variable est la même entre la MN et la MDD. On peut cependant supposer que les deux produits n'engendrent pas les mêmes coûts fixes. Par exemple, les producteurs de MN ont d'importants budgets publicitaires contrairement aux distributeurs. Cela pourrait se traduire par un coût fixe plus important 
associé à la MN par rapport à la MDD. Dans ce cas, les prédictions des deux modèles diffèrent. Ainsi dans le modèle de Mills, la MDD développée sera peu différenciée de la MN alors que dans le modèle de BMR, au contraire, le distributeur introduit une MDD significativement différenciée.

Jusqu’à présent on a supposé implicitement que le distributeur pouvait mettre en rayon autant de produits qu'il le désirait. Toutefois en pratique le linéaire est une ressource limitée. D’une part, au sein d'un magasin le distributeur doit allouer l'espace disponible entre les différentes catégories de produits. D'autre part, l'implantation ou l'extension de nouveaux magasins est, en France, strictement réglementée (Loi Raffarin). Dans un cadre d'analyse où le volume des ventes est contraint, le distributeur arbitre en faveur des produits qui dégagent les marges les plus importantes. Ces marges résultent de l'élasticité de la demande et des coûts d'approvisionnement. Lorsque les produits de haute qualité génèrent des marges plus importantes que ceux de basse qualité, la contrainte de capacité conduit le distributeur à privilégier les MN au détriment des MDD comme c'est le cas dans le modèle de Allain et Flochel (2001). Toutefois, dans un contexte de concurrence entre distributeurs où certaines MN sont utilisées comme produits d'appel, il est possible que les marges réalisées sur les MDD soient plus importantes que celles sur les MN ce qui conduit alors les distributeurs à favoriser les MDD sur le linéaire.

Les travaux théoriques précédents concluent à une baisse des prix des MN consécutive à l'entrée d'une MDD. Or les travaux empiriques montrent que c'est loin d'être systématiquement le cas. Certains auteurs ont donc cherché à développer un cadre d'analyse permettant d'expliquer pourquoi le prix des MN augmenterait avec l'introduction des MDD. Ainsi, Gabrielsen et Sørgard (2006) développent l'idée qu'après l'introduction de la MDD, la MN se recentrera sur une partie des consommateurs dont la demande est peu sensible au prix. Ainsi, l'introduction de la MDD permettra au distributeur de mieux discriminer entre deux types de consommateurs.

Plutôt que de considérer un continuum de consommateurs valorisant plus ou moins intensivement la 'qualité', comme cela est fait dans les autres travaux, Gabrielsen et Sørgard (2006) distinguent deux types de consommateurs : une partie des consommateurs ne considère que la $\mathrm{MN}$ alors qu'une autre partie des consommateurs arbitre entre MN et MDD lorsque celle-ci est présente sur le marché. Naturellement, l'impact de l'introduction de la MDD sur le prix de la MN dépend de la proportion des deux types de consommateurs :

- Lorsque la proportion des consommateurs loyaux est faible, c'est-à-dire que la MN fait face à une demande élastique, le producteur de MN a un comportement 'agressif' vis à vis de l'entrée de la MDD. En effet, celui-ci pratique une stratégie de prix limite qui dissuade l'entrée de la MDD. Cette stratégie le conduit à baisser son prix de gros pour que le distributeur réalise suffisamment de profit sur la seule MN. Le prix de gros et le prix de détail de la MN diminuent. 
- Au-delà d'une certaine proportion de consommateurs loyaux cette politique de prix limite est trop coûteuse. Le producteur de MN préfère alors s'accommoder de l'entrée de la MDD en adoptant une stratégie de repli sur le segment des consommateurs loyaux. Dans ce cas, le prix de gros et le prix de détail de la MN augmentent en réponse à l'entrée effective de la MDD. En effet, avant l'introduction, la MN était tarifée en sorte que les deux types de consommateurs achètent le bien. Au contraire après l'introduction la MN se concentre sur son marché captif ce qui explique l'augmentation de son prix.

- Enfin lorsque la part des consommateurs loyaux est élevée, l'entrée de la MDD n’a pas d'influence sur le prix de la MN. En effet, avant l'entrée, seuls les consommateurs loyaux étaient servis. Les prix de gros et de détail étaient déjà au niveau du prix de réservation des consommateurs.

Ces résultats contrastent avec les résultats des modèles précédents. Ils montrent que les MDD peuvent être un outil aux mains des distributeurs pour mieux discriminer les consommateurs et mettent en évidence les implications potentielles de cette stratégie. En particulier, ils suggèrent que les MN peuvent trouver un intérêt à se concentrer sur les consommateurs les plus fidèles aux marques et dont la demande est donc plus inélastique.

Les modèles présentés jusqu'ici permettent également de montrer que le développement des MDD (ou la menace de développement) est un facteur qui augmente le pouvoir de négociation du distributeur si l'on considère le partage des profits au sein de la structure verticale. Ceci s'applique que la MDD soit effectivement introduite ou non.

\subsubsection{Les effets de l'introduction d'une MDD dans le cadre d'une tarification binôme}

Rey et Tirole (2000) ont analysé la forme des contrats entre producteurs et distributeurs. Si dans un premier temps les producteurs proposent aux distributeurs des prix linéaires dans les CGV (Conditions Générales de Vente), dans un second temps, le jeu de la coopération commerciale et des marges arrières attribuées de manière rétroactive fausse la linéarité des prix d’approvisionnement. Les auteurs suggèrent donc la possibilité d'interpréter les contrats entre producteurs et distributeurs en termes de tarifs binômes, où les montants de coopération commerciale servent à redistribuer les profits de l'amont vers l'aval (objectif de la franchise dans les modèles théoriques). ${ }^{18}$

Caprice (2000) étudie l'impact de l'introduction d'une MDD dans le cadre d'une tarification binôme entre le producteur amont et le distributeur. Il montre que le prix de la MN n'est pas affecté par l'entrée de la MDD. L'effet lié à la réduction de la double marginalisation disparaît ici car la tarification binôme permet justement d'éviter celle-ci. Le producteur amont tarifie au coût marginal son produit dans les deux situations. Par contre, la répartition du profit de la structure verticale est modifiée par l'introduction de la MDD. Grâce à la MDD, le distributeur est capable de capturer une 
partie plus importante du profit. Dans le cadre de ce modèle, comme le producteur est supposé avoir tout le pouvoir de négociation, le profit du distributeur est égal à son profit de réservation. En l'absence d'une MDD, le distributeur dégage alors un profit nul. Dès que le distributeur a une stratégie alternative (vendre la MDD), le producteur amont est obligé de laisser au distributeur une rente dont le montant est égal au profit que le distributeur ferait en ne distribuant que la MDD (i. e. son nouveau profit de réservation). Ainsi, le distributeur accroît son pouvoir de négociation en augmentant le profit qu'il peut obtenir en absence d'accord avec le producteur.

Les articles précédents, en supposant une situation initiale où seule la MN est disponible, concluent à un accroissement du surplus des consommateurs. Le gain de surplus provient de deux effets : un effet variété et un effet concurrence. Un effet variété car les consommateurs disposent après l'introduction d'un produit supplémentaire. Un effet prix car l'introduction (ou la menace d'introduction) aboutit à une baisse du prix de gros de la MN et à une baisse du prix final. Cependant, dans le cas où la MDD remplacerait une marque (secondaire) déjà existante, cet effet variété serait amoindri. Ainsi, si l'on suppose que la situation initiale est constituée d'une offre de deux MN, le remplacement de la MN secondaire par une MDD réduit considérablement l'effet variété dans l'accroissement du surplus des consommateurs. Cela pose également la question de la robustesse des résultats de Gabrielsen et Sørgard (2006). En effet, chez ces auteurs, l'augmentation du prix de la MN (consécutif à l'introduction de la MDD) dépend directement de la possibilité de discriminer les consommateurs lorsque deux biens sont sur le marché. Il pourrait en effet exister un équilibre de départ où la MN principale ne servirait que les consommateurs loyaux et la MN secondaire servirait les autres consommateurs. L'introduction de la MDD, en remplacement de la MN secondaire, ne changerait pas cette possibilité de discrimination. Le prix de la MN n’augmenterait alors pas suite à l’introduction de la MDD.

Les travaux précédents ont donc considéré que la MDD était un bien supplémentaire à distribuer. En pratique, compte tenu des contraintes de 'capacité' des linéaires, il se peut que la MDD remplace en fait un autre produit. Scott-Morton et Zettelmeyer (2004) formalisent cette idée. Dans leur modèle, le distributeur doit arbitrer entre la distribution de deux MN concurrentes ou d'une MN et d'une MDD. Un premier producteur offre une marque nationale leader qui sera distribuée de toute façon. Le distributeur, compte tenu de la restriction sur le nombre de produits qu'il peut vendre doit choisir entre distribuer une deuxième marque nationale ou une MDD. Les gains de la filière sont partagés entre les producteurs et le distributeur. Chaque producteur gagne une proportion donnée, fonction de son pouvoir de négociation, du profit incrémental de son produit. ${ }^{19}$ Les biens offerts sont différenciés selon deux caractéristiques. Tout d'abord, les MN ont une qualité supérieure à la MDD. Ensuite, les biens peuvent être positionnés pour satisfaire les goûts de l'un ou l'autre de deux types de consommateurs définissant ainsi deux segments de marché. Ainsi les consommateurs du premier (second) segment sont prêts à payer plus pour une MN positionnée sur le premier (second) segment que pour une MN positionnée sur le deuxième (premier) segment. Sachant que la MN leader est positionnée sur le 
segment le plus large (segment 1), la question posée est : le distributeur a-t-il intérêt à distribuer une seconde MN ou une MDD. L'introduction de la MDD, comparativement à l'introduction de la seconde $\mathrm{MN}$, conduit à un profit total de la filière plus faible. Mais elle conduit également à diminuer la contribution incrémentale de la MN leader. La part de surplus qui revient au distributeur sera donc plus grande. Le distributeur doit donc arbitrer entre augmenter la taille du profit à se partager ou augmenter la part du profit qui lui revient malgré un profit total plus faible. Lorsqu'il dispose d'un 'faible' pouvoir de négociation, il préfère introduire la MDD. Inversement, s'il dispose d'un pouvoir de négociation suffisamment important, il préfère introduire la seconde MN qui permet d'augmenter le profit de la filière. En effet, plus le pouvoir de négociation du distributeur est important, plus il est sensible au profit total de la structure verticale.

Ainsi, l'introduction de la MDD s'accompagne toujours d'une augmentation du profit du distributeur (sinon il n'introduirait pas celle-ci) et d'une diminution du profit du producteur de la MN (sauf dans un cas où il resterait constant). Le distributeur parvient à capter une part plus importante des profits de la structure verticale. En général, le profit de la structure verticale augmente et ceci pour deux raisons : d'une part une réduction de la double marginalisation qui préexistait (cas des modèles de Mills, 1995 et de Bontems et al, 1999) et d'autre part en raison de l'introduction d'un deuxième bien qui permet de discriminer la demande (tous les modèles). Néanmoins, lorsque la MDD remplace une autre marque, l'augmentation du profit global n'est plus forcément assurée. Les consommateurs bénéficient généralement de l'introduction des MDD pour les mêmes raisons : diminution de la double marginalisation dans certains cas et éventuellement accroissement du nombre de biens offerts. Nous résumons dans le Tableau 4 les principaux effets économiques de l'introduction effective d'une MDD.

Tableau 4 : Effet de l'introduction effective d'une MDD

\begin{tabular}{|l|c|c|c|c|c|c|}
\hline & Mills & BMR & Caprice & SMZ & \multicolumn{2}{|c|}{ Gabrielsen et Sørgard } \\
& & & & & A & B \\
\hline Prix de gros MN & - & - & $=$ & $=$ & + & $\mathrm{np}$ \\
\hline Prime fixe producteur & $\mathrm{np}$ & $\mathrm{np}$ & - & - & np \\
\hline Prix détail MN & - & - & $=$ & - & - & $=$ \\
\hline Profit MN & - & - & - & - & + & + \\
\hline Profit distributeur & + & + & + & + & + & + \\
\hline Profit structure & + & + & + & - & + & + \\
\hline Surplus Consommateurs & + & + & + & $/$ & + & + \\
\hline
\end{tabular}

BMR : Bontems, Monier et Réquillart ; SMZ : Scott-Morton et Zettelmeyer ;

(1) Dans SMZ, la MDD se substitue à une MN secondaire ; dans les autres modèles la MDD est un produit supplémentaire. np : non pertinent dans ce modèle

A : proportion de consommateurs loyaux ni trop faible ni trop élevée ; B : proportion de consommateurs loyaux élevée

\subsubsection{Les faits confirment-ils les prédictions des modèles?}


Plusieurs travaux empiriques récents étudient pour un nombre important de produits la variation du prix des MN en fonction du développement des MDD. Un premier groupe de travaux utilise des données temporelles agrégées issues de panel de consommateurs. Ces données permettent d'analyser les corrélations entre prix des MN et développement des MDD. Un deuxième groupe de travaux utilise des données temporelles issues de panel de distributeurs. Elles permettent de suivre l'évolution du prix des MN en fonction de la présence ou non de MDD dans un magasin donné.

Ainsi Ward et al. (2002) étudient l'impact du développement des MDD aux États-Unis. Ils utilisent des donnés mensuelles sur les prix, parts de marché et dépenses publicitaires pour 34 catégories de produits. Pour chacune de ces 34 catégories considérées, ils analysent la réponse des MN (en matière de prix, de promotions et de politique de différenciation) au développement des MDD. ${ }^{20}$ Ils montrent que l'accroissement de la part de marché des MDD s’accompagne :

i) d'un accroissement du prix des MN (ou sinon pas d'effet) ;

ii) d'une diminution du prix des MDD (ou sinon pas d'effet) ;

iii) d’un effet négatif ou nul sur le niveau général des prix.

Ils montrent également qu'un accroissement des parts de marché des MDD s'accompagne d'une diminution des activités promotionnelles des MN, qu'il s'agisse de promotions par les prix ou d'autres moyens.

Bontemps et al. (2005, 2006) étudient l'impact du développement des MDD en France. Ils utilisent des données mensuelles sur les prix et parts de marché pour un grand nombre de produits (218 catégories de produits). ${ }^{21}$ Leurs résultats vont dans le même sens que les résultats précédents. Lorsqu'il est significatif, un accroissement de la part de marché des MDD s'accompagne d'un accroissement du prix des MN (dans 90\% des cas). Ils montrent également que l'effet est différent selon le type de MDD considérée. Ils distinguent ainsi les MDD proprement dites des marques de maxidiscompte (Hard Discount) et des Premiers Prix. Ils montrent que l'accroissement de la part de marché des MDD (au sens strict) s'accompagne d'un accroissement du prix des MN plus important que celui résultant d’un accroissement de la part de marché des autres types de marques analysées.

Gabrielsen, Steen et Sørgard (2002), étudient l'impact de l'introduction des MDD en Norvège. Ils utilisent des donnés hebdomadaires sur les prix et les parts de marché pour 83 produits d'un panel de distributeurs. Pour chacune des catégories considérées, ils analysent l'évolution du prix des MN dans le temps. Ils ne retiennent dans l'analyse que les marchés pour lesquels l'introduction d'une MDD intervient au cours de la période d'observation, ce qui leur permet de distinguer une période avant entrée et une période après entrée. ${ }^{22}$ Lorsque l'effet de l'introduction des MDD est significatif sur l'évolution du prix de la MN (17 cas sur 83), l’impact est généralement positif (15 cas). Ainsi, l'introduction d'une MDD conduit à une augmentation du prix de la MN. L'augmentation du prix semble d'autant plus forte que la part de marché de la MDD n’est pas trop élevée. Enfin leurs résultats 
suggèrent que les réactions en prix (augmentation du prix) des MN sont plus importantes pour les marques leaders et largement distribuées que pour les autres.

Bonfrer et Chintagunta (2004) étudient également l'impact de l'entrée des MDD sur le prix des MN. Comme dans le travail précédent, ils utilisent des données issues d'un panel de magasins. Leur travail porte sur 35 catégories de produits. Dans un peu plus de la moitié des cas (19 cas sur 35 produits étudiés), ils montrent que l'entrée des MDD s'accompagne d'une augmentation du prix des MN. Inversement, dans les autres cas, l'effet est négatif. ${ }^{23}$

Ainsi la plupart de ces travaux empiriques réalisés sur un grand nombre de catégories de produits montrent que l'entrée et le développement des MDD s'accompagnent généralement d'une augmentation du prix des MN contrastant ainsi avec les conclusions des modèles théoriques. ${ }^{24}$

\subsection{Les MDD comme élément de différenciation entre distributeurs}

Jusqu’à présent, nous nous sommes focalisés sur la concurrence entre les produits au sein d’un même distributeur, et les conséquences de la rivalité MN-MDD dans le partage de surplus entre le(s) producteur(s) amont et le distributeur en aval. La partie qui suit s'intéresse au rôle des marques de distributeurs dans la concurrence entre détaillants. Seul un nombre limité de travaux relâchent l'hypothèse d'un distributeur en monopole sur la demande des consommateurs.

Dans ce cadre, les MDD sont considérées comme un élément permettant aux distributeurs de se différencier. Etant par définition des produits spécifiques aux enseignes, l’offre de produits ne sera pas identique entre les différentes enseignes. Par exemple, dans l'agro-alimentaire où les accords d'exclusivité sont rares, en l'absence de MDD les distributeurs vendent souvent les mêmes marques. De ce point de vue, ils ne sont pas différenciés. L’introduction des MDD ajoute donc un élément de différenciation entre détaillants ce qui est susceptible d'atténuer la concurrence entre eux. Un éventail de stratégies est utilisé par les enseignes. Certaines enseignes, comme Intermarché, développent des marques propres par catégorie de produits sans que le nom de l'enseigne apparaisse. D’autres, comme Carrefour, ont choisi d'apposer leur nom sur leurs produits, tout au moins en ce qui concerne les MDD

'standard' ${ }^{25}$ Enfin, certaines ont choisi des stratégies intermédiaires qui combinent ces deux aspects. Ces stratégies de signalisation évoluent. Ainsi, en 1999, Auchan a abandonné son choix initial de développement de marques propres par catégorie de produits pour une stratégie basée sur le nom de l'enseigne. Les implications de ces choix n'ont pas fait l’objet d'analyse économique précise. En revanche, l'idée plus générale que la MDD est un moyen de différenciation entre enseignes fait l'objet de quelques travaux qui rentrent dans le cadre de la concurrence en gammes entre distributeurs. On sait que lorsque la concurrence en prix est vive, des distributeurs rivaux ont intérêt à offrir une gamme différenciée afin d'éviter une offre symétrique multi-produits (Champsaur et Rochet (1989)). 
Avenel et Caprice (2006) étudient dans le cas d'une concurrence à la Cournot le choix de gamme de deux distributeurs. Ces distributeurs peuvent s'approvisionner en un bien de haute qualité auprès d'un monopole amont et en un bien de basse qualité auprès d'une frange concurrentielle. Pour des valeurs raisonnables des paramètres (en coût et qualité), un des deux distributeurs a intérêt à signer un accord exclusif (ce qui se traduit par ne pas commercialiser de qualité basse) avec le producteur de marque. Dans ce cas, les gammes sont différenciées puisque un distributeur propose une MN et l'autre propose une marque de moindre qualité acquise auprès d'une frange concurrentielle, ce qui peut s'interpréter comme une MDD. En effet, grâce au contrat exclusif, le producteur de marque peut proposer un prix de gros suffisamment faible pour s'assurer que le détaillant choisi sera assez agressif vis-à-vis de son concurrent.

La littérature en marketing s'est évidemment intéressée au rôle des MDD dans la concurrence entre distributeurs. En particulier, les relations entre achat de MDD et fidélité à l'enseigne sont au cœur de l'analyse. Comme le résument Sudhir et Talukdar (2004), si les MDD sont utilisées comme outil de différenciation par les distributeurs, il est possible qu'un consommateur qui achète une MDD d'une enseigne donnée réalise dans cette enseigne une fraction plus importante de ses achats. Cependant les MDD peuvent également attirer les consommateurs les plus sensibles aux prix. Dans ce cas, l'achat de MDD n'impliquera pas que le consommateur réalise une part plus grande de ses achats dans l'enseigne. Sudhir et Talukdar (2004) étudient cette question à partir des données d'achat sur 44 catégories de produits par 2000 ménages fréquentant un distributeur donné. Ils montrent que les ventes globales (du magasin) diminuent avec l'augmentation de la part de marché des MDD, inversement les profits augmentent. Ils montrent également que l'achat d'une large gamme de MDD a un effet positif sur les ventes et les profits. L'augmentation de profit en dépit de la concurrence entre distributeurs résulterait donc d'un effort de différenciation de gammes. Ces résultats suggèrent donc que les MDD contribuent à la différenciation entre les enseignes plutôt qu’à une sensibilité accrue aux prix.

Jusqu’alors nous avons considéré la MDD comme un nouveau produit, de qualité exogène inférieure à celui d'une MN, que le distributeur obtenait au coût marginal. Dans ce cadre, une MDD n'est pas vraiment différente d'un produit de qualité basse (par exemple, une marque sans notoriété) produite par une petite firme. Une caractéristique essentielle de la MDD réside dans le choix par le distributeur des caractéristiques du produit. ${ }^{26}$ Ce choix est stratégique car le distributeur positionne ce produit pour en tirer le profit le plus grand possible. Ce choix sera notamment guidé par un arbitrage entre un souci de discrimination de la demande et un souci d’accroître le pouvoir de négociation. 


\subsection{Le positionnement stratégique des MDD}

Nous distinguons, comme dans les sections précédentes, les deux types possibles de tarifications des contrats (linéaires et binômes) car ils influent sur le choix stratégiques des caractéristiques de la MDD par les détaillants.

\section{Le positionnement de la MDD dans le cadre d'une tarification linéaire}

Dans le modèle de Bontems et al. (1999), on peut montrer que le choix du niveau de qualité du bien de qualité basse dépend de la structure de marché considérée et de l'acteur qui fixe le niveau de qualité du bien. ${ }^{28}$ Les quatre situations suivantes ont été classées selon l'ordre croissant du niveau de la qualité basse :

1. Monopole amont multiproduit qui choisit la qualité ;

2. Duopole amont, le producteur de basse qualité choisit la qualité ;

3. Monopole amont en haute qualité, industrie compétitive fournissant la basse qualité, le distributeur choisit le niveau de qualité basse ;

4. Duopole amont, le distributeur choisit le niveau de la qualité basse. ${ }^{29}$

Ainsi, lorsque le distributeur choisit la qualité du bien de basse qualité, il définit une qualité supérieure à celle qui serait fixée par un producteur (comparaisons des cas 1 et 3 d'une part et des cas 2 et 4 d'autre part). En effet, le distributeur utilise la MDD comme outil pour réduire le prix de gros de la MN. Ceci l'incite à réduire la différenciation entre MN et MDD. Un producteur amont n’a pas cette incitation et choisit donc un produit plus différencié.

Sayman, Hoch et Raju (2002) analysent le choix en localisation de la MDD lorsque deux marques nationales sont déjà présentes chez un distributeur. Selon leur analyse, le distributeur a intérêt à positionner sa marque proche de la MN leader, car même si cela fait chuter la demande de cette dernière (en raison de la concurrence), le distributeur obtient une diminution significative sur le prix de gros de la MN. Ce gain en pouvoir de négociation par le distributeur est d'autant plus important que la marque leader a une part de marché importante. Récemment, Du, Lee et Staelin (2006) montrent que le choix du positionnement de la MDD près de la MN leader n'est pas systématique. Ils développent un modèle de demande plus riche que le précédent qui inclut différenciation verticale et horizontale. Dans un nombre important de situations (définies par l'écart de qualité entre les deux MN et l'importance de la différenciation horizontale), ils montrent que le distributeur a intérêt à introduire la MDD proche de la seconde MN ou dans une position intermédiaire entre les deux MN. 
Dans tous les cas, l'effet de réduction du phénomène de double marginalisation est un ingrédient important dans le choix de positionnement du produit. On peut donc se demander dans quelle mesure ces résultats sont robustes au choix du mode de tarification entre producteurs et distributeurs.

La faiblesse de considérer des contrats en prix linéaire tient au fait que le producteur ne peut jouer que sur le prix de gros pour atteindre deux objectifs différents : maximisation des profits et partage du surplus avec le détaillant. Lorsque les contrats sont des tarifs binômes, le parasitage entre les deux objectifs disparaît car la franchise, indépendante des quantités, permet de redistribuer les profits dans la structure verticale sans modifier les allocations optimales.

\section{Le positionnement de la MDD dans le cadre d'une tarification non-linéaire}

Caprice (2000), dans le cadre d'une tarification binôme, a également étudié le choix de la qualité optimale par le distributeur. On a vu (section 2.1) que le fait de pouvoir distribuer une MDD (un deuxième produit) permettait au distributeur de capter une partie plus importante du profit de la structure verticale. Caprice montre que la possibilité pour le producteur amont de s'engager sur les conditions du contrat qu'il propose au distributeur est cruciale dans le choix de qualité de la MDD par le distributeur.

Ainsi, si le producteur de MN peut s'engager sur un contrat de vente préalablement au choix de la qualité de la MDD par le distributeur, ce dernier choisit une qualité de la MDD qui maximise le surplus de la structure intégrée. En effet, dans le cadre de la tarification binôme, le producteur amont vend au coût marginal son produit de telle sorte que le distributeur se trouve dans la même position que la structure intégrée (monopole multi-produit). Au contraire, si le producteur ne peut s'engager sur un contrat de vente préalablement au choix de la qualité de la MDD, alors le distributeur choisira une qualité plus haute que précédemment. ${ }^{33}$ En effet, la qualité sera choisie de sorte qu’en cas de désaccord, i.e. non référencement de la MN, le profit du distributeur soit maximal. En d'autres termes, le distributeur choisira une qualité qui maximise son profit de réservation.

On notera que dans ce modèle, le distributeur obtient le même profit quelle que soit la possibilité d'engagement du producteur amont. En effet, dans cette formalisation, le profit du distributeur est égal à son profit de réservation qui correspond au profit qu'il gagnerait en cas d'échec de la négociation avec le producteur MN. C’est donc le profit provenant de la vente de la seule MDD. Or, dans les deux cas étudiés, la qualité de la MDD qui serait choisie par le distributeur en cas d'échec de la négociation est identique et correspond à la qualité qui maximise le profit du distributeur. Le producteur, quant à lui, préfère la situation où il peut s'engager sur le contrat avant le choix de qualité de la MDD par le distributeur. En effet, dans ce cas, le choix de qualité par le distributeur aboutit à un degré de différenciation entre les produits qui est optimal du point de la structure intégrée, et le profit global est alors plus élevé. 
Scott Morton et Zettelmeyer (2004) analysent également le choix de positionnement de la seconde MN ou de la MDD (voir §2.1.2 pour une présentation du modèle). Ils montrent que la seconde MN serait introduite sur le deuxième segment de consommateurs (i. e. celui que n’occupe pas la MN leader) puisque c'est finalement la seule façon pour elle de se différencier de la MN leader. Inversement, la MDD est introduite sur le premier segment de consommateurs (i.e. celui qu'occupe la MN leader) puisque l'objectif est de diminuer au maximum le gain incrémental de la MN leader.

Il apparaît une nouvelle fois que le choix des caractéristiques des biens est différent selon qu’il est décidé par un producteur amont ou par un distributeur. Outre un souci de discriminer entre les consommateurs, la MDD est également un outil dans les mains du distributeur pour s'accaparer une part plus importante du surplus de la filière. Même si dans le strict cadre de ce modèle, les choix de caractéristiques des biens sont limités (les qualités des biens sont fixes et exogènes), les mécanismes mis en œuvre montrent que la MDD est positionnée de façon à concurrencer fortement la MN alors que le producteur de la deuxième MN est moins enclin à le faire.

Bontems (2004) examine les conséquences en terme de bien-être de l'introduction d'une MDD sur un marché où un distributeur en position de monopole écoule deux produits substituts quelque soit la structure de marché amont et où le positionnement de tous les produits (MN ou MDD) est endogène. La structure de marché amont varie entre le cas 1 de la section précédente (monopole amont multiproduit), le cas 2 (duopole amont de $\mathrm{MN}$ ) et le cas 3 (monopole amont monoproduit et frange concurrentielle fabricant une MDD pour le compte du distributeur). Ceci permet par comparaison d'estimer les gains potentiels réels de bien-être lors de l'introduction des MDD, en neutralisant l'effet positif sur le surplus des consommateurs lié à l'ajout d'un produit supplémentaire puisque dans tous les cas les consommateurs ont le choix entre 2 produits. En supposant que le ou les fabricants en amont peuvent proposer des contrats à quantité fixée ou des tarifs binômes à prendre ou à laisser, l'auteur montre que l'introduction d'une MDD en lieu et place d'une MN peut parfois non seulement diminuer le surplus des consommateurs mais aussi le bien-être, dans le cadre d'un modèle standard de différenciation verticale. En effet, l'opportunisme du distributeur conduit à un positionnement de la MDD de manière à maximiser son profit de réservation lors de la négociation avec le producteur amont. Cette stratégie conduit alors à « cannibaliser » de manière excessive (du point de l'industrie) les ventes de la MN. En revanche, dans le cas d'un duopole en MN, le positionnement des produits (ainsi que leurs quantités) est optimal du point de vue de l'industrie (à coup sûr dans le cas de contrat imposant des quantités fixées et sous certaines conditions dans le cas de tarifs binômes) conduisant alors à la maximisation du profit joint. Toutefois, de manière paradoxale, le surplus total des consommateurs est plus faible dans le cas 3 que dans le cas 2 alors même que le profit joint de l'industrie n'est pas maximal dans le cas 3. Du point de vue du bien-être, l'accroissement de profit du distributeur offrant une MDD ne suffit pourtant pas à compenser la diminution de profit du secteur amont et la diminution de surplus des consommateurs. 
Les faits confirment-ils les prédictions des modèles ?

Mesurer la 'qualité' des MDD par rapport aux MN est difficile. D'une part, il est difficile d'établir l'évolution des caractéristiques des produits, d'autre part cette notion a un caractère subjectif important. Ceci explique le fait que relativement peu de travaux analysent les choix de qualité des produits MN ou MDD et leur évolution.

Putsis (1997) étudie pour un grand nombre de catégories de produits et un grand nombre de marchés locaux (données en coupe), les fonctions de réaction en prix des MN et des MDD. Ses résultats suggèrent qu'une part de marché des MDD plus élevée entraîne une baisse du prix des MN et une augmentation du prix de la MDD. Ce lien négatif entre part de marché des MDD et écart de prix entre MN et MDD est pour le moins contre intuitif. Si l'on admet qu'un des éléments importants qui déterminent la concurrence entre MN et MDD est le niveau de qualité relatif entre MN et MDD, ce résultat pourrait s'expliquer par le biais de cette variable non observée. ${ }^{36}$ Une part de marché plus élevée de la MDD serait alors expliquée par un niveau plus élevé de qualité de la MDD.

Un certain nombre d'auteurs ont néanmoins analysé le choix de positionnement des MDD. Ainsi, Sayman et al. (2002) montrent que lorsque les MDD suivent une politique de ciblage par rapport aux marques nationales, elles le font en priorité en direction des marques nationales les plus fortes. Scott-Morton et Zettelmeyer (2004) montrent également qu'une proportion importante de MDD cible directement la principale MN (ce qui correspond bien au développement des produits appelés me-too). Les résultats de Bontemps et al. (2006) obtenus sur 21 groupes de produits sont cohérents avec les conclusions précédentes. D’une part le développement des MDD semble s'être fait majoritairement au détriment de la MN principale, et d'autre part le prix des MN secondaires est moins affecté par le développement des MDD que celui de la MN principale. Le distributeur en développant une MDD proche de la MN principale peut mettre en œuvre une politique de segmentation en choisissant un écart de prix significatif entre les deux produits menant ainsi une stratégie de discrimination de la demande.

\subsection{MDD et réputation d'enseigne}

Les MDD sont un des éléments qui permettent aux distributeurs de construire une image d'enseigne. Pour aborder cette question, il est nécessaire de développer une analyse dynamique où le choix des consommateurs dépend des achats passés donnant alors du sens à la notion de réputation. Les travaux présentés jusqu'ici considéraient les biens (MN ou MDD) comme des biens de recherche où la qualité est connue avant consommation et ne permettaient donc pas de traiter la question de réputation. C’est pourquoi certains auteurs considèrent la MDD comme un bien d'expérience. BergèsSennou et Waterson (2005) étudient une simple structure verticale où un producteur de MN connue (la MN est alors considérée comme un bien de recherche) vend son bien à un distributeur. Le distributeur doit décider s'il veut introduire une MDD (considérée comme un bien d'expérience car c'est un 
nouveau produit) et si oui, quel niveau de qualité choisir. Dès lors, les consommateurs, ne découvrant la qualité qu'après achat et décidant d'acheter sur la base d'anticipation, peuvent être trompés par le distributeur. Ce dernier peut en effet faire croire que la MDD est de bonne qualité afin de récupérer des recettes en première période sans payer le coût de production associé à la qualité. Le détaillant perd cependant les consommateurs à la seconde période. Les auteurs montrent alors que le détaillant est d'autant plus incité à produire une MDD de bonne qualité, et donc maintenir sa réputation, que la fréquence d'achat du bien est importante. Cette incitation est d'autant plus forte que le pouvoir de négociation du distributeur est important. Les gains provenant de la vente de la MDD augmentant avec le pouvoir de négociation du distributeur, l'enjeu de maintenir la réputation est donc de plus en plus élevé.

Peu de travaux empiriques abordent cette question. Sethuraman et Cole (1997) montrent que la différence de consentement à payer entre MN et MDD décroît avec la fréquence d’achat du bien. Del Vecchio (2001), à partir de données déclaratives, constate que la qualité perçue de la MDD est d'autant plus faible que la fréquence d'achat est importante. Par ailleurs, les travaux de Sudhir et Talukdar (2004) montrent que les MDD sont un facteur de fidélité à l'enseigne dans la mesure où plus la part des MDD est importante pour un consommateur plus ses dépenses dans l'enseigne impliquée le sont également.

\section{Quels sont les enjeux de la production des MDD pour les firmes de l'amont?}

Les MDD sont produites par différents types de firmes. Selon PLMA, les producteurs de MDD sont de trois types :

- Des grandes firmes qui produisent à la fois leurs MN et des MDD pour un ou plusieurs distributeurs ;

- Des petites et moyennes entreprises qui se spécialisent dans la production de biens particuliers et concentrent leur production sur les MDD ;

- Des distributeurs ou grossistes qui possèdent leurs propres usines de production et fabriquent leur MDD pour leurs magasins (intégration verticale).

Si l'on prend l'exemple des produits laitiers, on retrouve des firmes appartenant à chacune de ces catégories. Ainsi, la plupart des grandes firmes laitières produisent des MDD (Bongrain, Lactalis, Nestlé, SODIAAL, Entremont). Des PME se sont spécialisées, au moins un temps, dans la production de MDD (Senoble qui produisait essentiellement des MDD et qui a maintenant créé ses propres marques de produits). Enfin, au moins un distributeur possède un site de production (Intermarché qui possède l'unité de production de St Père). Galizzi et al. (1997) montrent que pour l’Italie, où les parts 
de marchés des MDD sont faibles, les distributeurs confient dans un premier temps la production de leurs marques à des PME. Les auteurs prédisent qu'à la fin de ce stade initial du développement des MDD, les producteurs de grandes marques fabriqueront également les produits des distributeurs.

Hormis ces éléments descriptifs, peu de travaux économiques ont été consacrés à l'étude de cette question. Nous présentons ici quelques pistes de recherche. Les MDD étant considérées comme un produit concurrent des MN, a priori, les producteurs de MN perdent des bénéfices lorsqu'un distributeur introduit sa MDD. Ce n'est pourtant pas si simple. S'il est vrai qu'au final, MDD et MN se concurrencent sur le marché aval, il reste tout de même l'enjeu de la production de la MDD. Sur ce point, les producteurs MN peuvent néanmoins obtenir des profits additionnels.

Un argument souvent avancé par les producteurs aux marques bien établies est que la production de MDD leur permet d'utiliser des capacités de production excédentaires. Un argument également cité par Hoch (1996). Certes, ces producteurs vont alors produire un bien qui leur fera directement concurrence, mais si eux ne le produisaient pas, d'autres le feraient. Les revenus supplémentaires venant de la production de la MDD iraient alors à d'autres firmes. Ce raisonnement admet cependant une limite : il suppose implicitement que les entreprises isolées susceptibles d'être choisies pour produire la MDD soient capables de fabriquer un produit aux caractéristiques proches de celui du producteur de MN. Si tel n'est pas le cas, un producteur de MN peut ne pas avoir intérêt à produire la MDD car il n’y aurait alors pas d'alternative crédible pour le distributeur de trouver un concurrent "sérieux ». C'est le cas de Coca-Cola qui possède une assise de marque telle qu'aucun concurrent MDD ne le gène vraiment. Aussi, jusqu'alors Coca-Cola ne produit pas de MDD.

Lorsque l'alternative de production d'une MDD proche de la MN existe, le producteur peut donc avoir intérêt à produire son propre produit concurrent. Encore faut-il que le distributeur le lui demande. En effet, le distributeur est alors face à un arbitrage entre gain d'efficacité et rente de répartition. D’un côté le producteur de la MN, s'il produit la MDD, aura des coûts de production plus faible qu'une firme isolée issue d'une frange concurrentielle entrant. ${ }^{39}$ D’un autre côté, en confiant sa MDD au producteur de la MN, le distributeur donne les deux produits dont dépendent majoritairement son profit à la même firme : le producteur de la MN. Ceci va donc avoir une influence sur les conditions tarifaires de la MN.

Comme le montre Bergès-Sennou (2006), lorsque le pouvoir de négociation du distributeur face au producteur de MN est faible, le distributeur préférera confier sa MDD à une entreprise indépendante. En effet, si le distributeur confie la MDD au producteur de la MN, il doit également partager avec ce dernier les gains qu'il fait sur la MDD. Or si son pouvoir de négociation est faible, il bénéficierait peu des gains en efficacité apportés par une production de la MDD par le producteur de MN. Il préfère donc confier la production de la MDD à la firme isolée. Un second résultat est que plus la loyauté des consommateurs envers la MN est élevée, plus le distributeur aura intérêt à confier sa MDD au producteur MN. Une fidélité des consommateurs élevée pour la MN signifie que la MDD n'est pas une véritable alternative c'est-à-dire que cette dernière n’engendre pas pour le distributeur 
des profits de réservation élevés lors de la négociation. Dès lors, mieux vaut simplement profiter de l'avantage en coût du producteur MN plutôt que de maintenir une production indépendante mais coûteuse de la MDD.

\section{Quelques conclusions}

A court terme, la plupart des modèles présentés concluent à l'effet positif sur le bien-être de l'introduction des MDD. Dans ces modèles, la MDD est le plus souvent considérée comme un bien supplémentaire qui permet au distributeur d'augmenter ses profits au détriment de l'amont soit en pesant sur les prix de gros soit en accroissant sa part des gains au sein de la filière. Dans ces modèles, le consommateur bénéficie d'une part d'un accroissement du nombre des biens disponibles et d'autre part de l'effet positif de concurrence entre MN et MDD.

Si l'on considère qu'en pratique les MDD viennent en substitution de marques 'secondaires' et non pas en produits additionnels, l'effet bénéfique lié à l'augmentation du nombre de 'qualités' disponibles pour le consommateur disparaît. Cela étant, on a vu que les choix de qualité opérés par un producteur ou par un distributeur n'étaient pas identiques. La MDD étant généralement de 'qualité' plus proche de la MN, le consommateur bénéficiera d'une concurrence accrue entre les produits mais inversement il pourra pâtir de la moindre différenciation des biens auxquels il fait face. Ainsi dans ce cadre peut-être plus réaliste, il n'est pas sûr que l'introduction d'une MDD aboutisse à un gain de bien-être. A long terme, le développement des MDD peut également modifier la concurrence entre distributeurs. Ainsi, la présence des MDD en rendant les distributeurs plus différenciés peut diminuer l’intensité de la concurrence en prix entre eux. Un tel effet serait négatif sur le bien-être.

A plus long terme, l'impact des MDD pourrait être moins positif même si ce point n'a pas fait l'objet de travaux spécifiques. En effet, le développement des MDD conduit à un partage différent des profits au sein des structures verticales producteurs-distributeurs. La diminution des profits de l'amont peut conduire celui-ci à moins innover et à terme réduire les choix des consommateurs. Cela est renforcé par la stratégie observée de mise en vente de produits 'me-too' qui sont une forme de freeriding sur les dépenses de recherche et développement de nouveaux produits qui est susceptible de décourager sur le long terme les efforts de mise au point de nouveaux produits.

Même si le développement des MDD a fait l'objet de recherches théoriques et empiriques depuis quelques années, un certain nombre de questions restent ouvertes. Alors que la plupart des modèles théoriques concluent à une diminution du prix des marques nationales en réponse au développement des marques de distributeurs, les travaux empiriques récents mettent en évidence un effet inverse. Si ces résultats empiriques étaient confirmés par d'autres études (par exemple sur d'autres pays), il deviendra particulièrement nécessaire d'en comprendre la raison et cela nécessitera le développement de modèles théoriques alternatifs à ceux développés jusqu’alors. D’une part, il sera important de s'affranchir de l'hypothèse forte de tarification linéaire qui, compte tenu de l'existence de 
primes fixes, n'est pas totalement adaptée ${ }^{43}$; d'autre part les MDD pourraient être considérées plus comme des produits venant se substituer à d'autres et non comme des produits supplémentaires. La prise en compte du fait que nombre de producteurs de MN produisent également des MDD est une autre piste à considérer. Le producteur cherchant alors à mieux discriminer la demande.

Par ailleurs, la plupart des modèles développés considèrent les relations entre un producteur et un distributeur. Mieux intégrer l'impact des MDD sur la concurrence entre distributeurs reste donc une question relativement peu développée en raison notamment de la complexité des équilibres en gamme.

Sur le plan empirique, une meilleure compréhension des choix de marques par les consommateurs est nécessaire et ceci pour mieux évaluer l'impact de la fidélité des consommateurs aux marques. De plus, la prise en compte de la 'qualité' des MDD (par rapport à celles des MN) reste un point important mais difficile à mener compte tenu des informations disponibles.

Le développement des travaux empiriques prenant explicitement les comportements de négociation entre producteurs et distributeurs est une piste prometteuse pour mieux intégrer les stratégies économiques dans les analyses empiriques. 


\section{Références}

Allain M.L. et L. Flochel, 2001. Marques de distributeurs et contraintes de capacité. Revue économique, 52(3):643-653.

Avenel E. et S. Caprice, 2006. Upstream Market Power and product line differentiation in retailing, International Journal of Industrial Organization, 24: 319-334.

Baroux C., 2000. Deux siècles de bras de fer entre l'industrie et le commerce. Les Echos, 05/01/2000.

Bergès-Sennou F., Bontems P. et V. Réquillart, 2004, Economics of Private labels: a survey of the literature. Journal of Agricultural and Food Industrial Organization, 2(1), article 3, 25 pages ;

Bergès-Sennou F. et M. Waterson, 2005, Private labels as experience goods, Journal of Agricultural and Food Industrial Organization, 3(2), article 9, 18 pages ;

Bergès-Sennou F. ,2006, Store loyalty, bargaining power and the private label production issue, European Review of Agricultural Economic, Vol. 33(3), p. 315-335 ;

Bonfrer A. et P. Chintagunta, 2004. Store Brands: Who Buys Them and What Happens to Retail Prices When They Are Introduced?, Review of Industrial Organization, Vol. 24, p. 195 - 218.

Bonnet C. et P. Dubois, 2006. Non linear Contracting and endogenous Market Power in Manufacturers and Retailers Relationships, Document de travail INRA-ESR, GREMAQ, Toulouse.

Bonnet C., Dubois P., et M. Simioni, 2005. Manufacturers and Retailers Strategic Relationships: Empirical Tests on Differentiated Products Markets, INRA-ESR, GREMAQ, Toulouse ;

Bontemps, C., Orozco, V., Réquillart, V. et A. Trévisiol, 2005. Price Effects of Private Label Development. Journal of Agricultural and Food Industrial Organization, Volume 3, Article 3.

Bontemps, C., Orozco, et V., Réquillart, 2006. Private Labels, National Brands and Food prices, IDEI Working paper, $\mathrm{n}^{\circ} 402$.

Bontems P., Monier S. et V. Réquillart, 1999. Strategic effects of private labels. European Review of Agricultural Economics, 26(2) :147-165.

Bontems P., 2004. On the welfare impact of store brands, Document de recherche INRA-Toulouse, 20 pages ;

Caprice S., 2000. Contributions à l'analyse de la puissance d'achat dans les relations verticales : interactions stratégiques et marques de distributeur, Thèse Université Paris I, 280 pages.

Champsaur P. et J.C. Rochet, 1989. Multiproduct duopolists, Econometrica, 57(3) :533-557.

Chardon O. et Dumartin S., 1998. Fidélité aux enseignes, fidélité aux marques :le choix des consommateurs. INSEE Première, $n^{\circ} 609$.

Chintagunta P.K., Bonfrer A. et Song I., 2001. Investigating the effects of store brand introduction on retailer demand and pricing behavior. Working paper, Graduate school of business, University of Chicago. 
Cotterill R.W., Putsis W.P. and R. Dhar, 2000. Assessing the competitive interaction between private labels and national brands, Journal of Business, 73(1):109-138.

Dhar S. K. et Hoch S. , 1997, Why Store Brand Penetration Varies by Retailer, Marketing Science, 16(3), p. 208-227 ;

DelVecchio D. 2001. "Consumer perceptions of private label quality: the role of product category characteristics and consumer use of heuristics", Journal of Retailing and Consumers Services, Vol. 8, p. 239 - 249.

Du R. , Lee E. et Staelin R. , 2006. Bridge, Focus, Attack or Stimulate: Retail Category Management Strategies with a Store Brand. Quantitative Marketing and Economics. Forthcoming.

Fournier, 1996. Les marques de distributeurs. Libre Service Actualités, 1472:10-15.

Gabrielsen T.S. et L. Sorgard, 2006. Private labels, price rivalry and public policy. European Economic Review. Forthcoming.

Gabrielsen T.S., Steen F. et L. Sorgard, 2002. Private labels entry as a competitive force ? An analysis of price responses in the Norwegian food sector. XIIth EARIE conference. Madrid.

Galizzi, L. Venturini et S. Boccaletti, 1997. Vertical relationships and dual branding strategies on the Italian Food Industry, Agribusiness, 13(2), p. 185-195.

Hoch S., 1996. How should national brands think about private labels? Sloan Management Review, 37(2):89-102.

Kapferer J.N., 1998. Les Marques : capital de l'entreprise, Editions d'Organisations, ISBN : 2-70812145-6, 576 pages.

Le Borgne C. 1996. La marque de distributeur : l'étude des relations fournisseur - distributeur, Mémoire de DESS en Droit des Affaires, Université de Bourgogne, 53 pages.

Mañez J.C. et M. Waterson, 2001. Multiproduct firms and product differentiation: a survey. University of Warwick Working Paper n 594, 56 pages.

McGoldrick P. , 1984, Grocery Generics - An extension of the Private Label Concept, European Journal of Marketing, 18(1), p.5 - 24 ;

Mills, D.E., 1995. Why retailers sell private labels. Journal of Economics and Management Strategy, 4(3) :509-528.

Office of Fair Trading, March 2006, Grocery Market: Proposed decision to make a market investigation reference, OFT 838, 67 pages ;

PLMA International Private Label Yearbook: A statistical guide to market share trends, Private Label Manufacturers Association and AC Nielsen, (2005). www.acnielsen.com

Putsis W.P., 1997. An empirical study of the effect of brand proliferation on private label-national brand pricing behavior. Review of Industrial Organization, 12 :355-371.

Rey Patrick et Tirole Jean (2000), Régulation des relations entre fournisseurs et distributeurs, Conseil d’Analyse Economique, 92 pages, Paris. www.cae.gouv.fr

Rey P., 1997. Impact des Accords Verticaux entre Producteurs et Distributeurs, Revue Française d'Économie, XII (2), p. 3-56. 
Sayman S., Hoch S.J. et J. Raju, 2002. Positioning of store brands. Marketing Science, 21(4), 378-397.

Scott Morton F. et F. Zettelmeyer, 2004. The strategic positioning of store brands in retailer manufacturer bargaining, Review of Industrial Organization, Vol. 24, p. 161-194.

Sethuraman R. and C. Cole, 1997, Why Do Consumers Pay More for National Brands than for Store Brands?, Working Paper, University of Iowa, 36 pages;

Steiner Robert L., 1985. The Nature of Vertical Restraints. The Antitrust Bulletin, Spring, 143-197.

Steiner Robert L., 2004. The Nature and Benefits of National Brand/Private Label Competition. Review of Industrial Organization, 24: 105-127.

Sudir K., et D. Talukdar, 2004. Does Store Brand Patronage Improve Store Patronage ?, Review of Industrial Organization, Vol. 24, p. 143-160.

Villas-Boas S.B., 2005. Vertical contracts between manufacturers and retailers: Inference with Limited Data. Working Paper 943R2, Department of Agricultural \& Resource Economics, University of California, Berkeley.

Ward M.B., Shimshack J.P., Perloff J.M., and M.J. Harris, 2002. Effects of the private-label invasion in food industries. American Journal of Agricultural Economics, 84(4):961-973.

Web sites:

Linéaires, www.linéaires.com

PLMA, www.plmainternational.com

${ }^{1}$ PLMA, voir www.plmainternational.com

${ }^{2}$ D’autres termes désignent également les MDD : marques propres (par exemple, Pâturages qui désigne les produits laitiers chez Intermarché), marques d'enseigne (par exemple : produits Carrefour), marques ombrelles (par exemple marque Repère chez Leclerc, Reflets de France chez Carrefour). La plupart des analyses économiques ne distinguent pas cette différence entre MDD car elles se focalisent sur le fait que le produit est spécifique ou non à un distributeur donné. Intégrer ces différences de MDD nécessiterait certainement une analyse en terme d'économie de gamme ou d'envergure et de lien perçu par les consommateurs entre produit et enseigne.

${ }^{3}$ Se référer à Mañez-Waterson (2001) pour une revue de littérature sur la concurrence entre firmes multiproduits.

${ }^{5}$ Les produits premiers prix sont généralement définis comme des produits dont le prix est inférieur à $80 \%$ du prix moyen des produits. Il s’agit souvent des produits d’entrée de gamme.

${ }^{6}$ Voir Kapferer (1998) pour des études marketing expliquant la disparité des parts de marché au sein des marques.

${ }^{7}$ Ainsi, Chardon et Dumartin (1998) montrent que les consommateurs sont plus enclins à changer de produits dans une enseigne plutôt qu’à changer d'enseigne s’ils ne trouvent pas le produit désiré. Cela confère donc une position de négociation favorable au distributeur.

${ }^{8}$ Cette idée est cohérente avec les résultats de Dhar et Hoch (1997) qui montrent qu'un petit nombre de producteurs de MN (au sein de la catégorie de produits étudiés) est un facteur favorisant le développement des MDD. En effet, plus le nombre de producteurs amont est réduit plus leur pouvoir de négociation est a priori 
élevé. Le distributeur est donc d'autant plus inciter à développer sa propre marque pour améliorer sa position de marchandage.

${ }^{9}$ Les résultats restent valables si le distributeur supporte un coût de distribution.

${ }^{10}$ Cette affirmation doit maintenant être nuancée avec l'apparition de MDD 'haut de gamme' tels que Reflets de France, Saveurs étrangères. La majorité des MDD se situe néanmoins à des niveaux de qualité perçus inférieur ou égal à celui des MN. Voir également Steiner (2004) pour des commentaires détaillés sur la différence de perception entre MN et MDD. Par ailleurs, une enquête de l'Insee (Chardon et Dumartin, 1998) fait apparaître que les consommateurs qui achètent les MDD considèrent le bon rapport 'qualité - prix' de ces produits comme étant l'avantage premier de ces biens.

${ }^{12}$ Cela renvoie par exemple à des coûts de publicité : les MN sont alors considérées par les consommateurs comme des biens de qualité supérieure en raison de la publicité dont elles font l'objet. Les dépenses de publicité sont indépendantes des volumes de vente et sont donc considérées comme des coûts fixes.

${ }^{13}$ Si la MDD a un avantage en coût sur la MN, pour des valeurs élevées de la qualité de la MDD, seule la MDD est sur le marché et la MN n’est plus référencée (Voir Allain et Flochel, 2001).

${ }^{14} \mathrm{Ne}$ sont pas compris les coûts fixes liés à la production des biens (non modélisés).

${ }^{15}$ Sous certaines conditions, le prix de la MN peut devenir croissant. En effet, l'augmentation de la qualité de la MDD a deux effets : d'une part, une plus forte concurrence qui a pour effet de faire baisser le prix de gros de la MN et d'autre part une augmentation du coût marginal de production qui agit en sens inverse. La somme des deux effets peut conduire à une augmentation du prix de la MN.

${ }^{16}$ L'hypothèse d'un coût de production de la MDD inférieure à celui de la MN (à qualité identique) aboutirait au même résultat : la MDD serait toujours introduite.

${ }^{17}$ Ce résultat est lié à la forme fonctionnelle de la courbe de coût utilisée (coût quadratique en l'occurrence).

${ }^{18}$ Une étude récente du marché de l'eau en bouteille pour la France de Bonnet, Dubois et Simioni (2005) montrent que les producteurs et les détaillants utilisent des contrats non linéaires plutôt qu’un simple prix de gros unitaire.

${ }^{19}$ Le profit incrémental d'un producteur est calculé comme étant égal à la différence entre le profit de la filière lorsque les deux produits sont distribués et le profit lorsque seul le produit concurrent est distribué.

${ }^{20}$ En pratique, pour les principales MN de chaque marché, le prix de la MN est régressé sur différentes variables explicatives dont la part de marché des MDD.

${ }^{21}$ Bontemps, Orozco et Réquillart (2006) généralisent à un grand nombre de produits le travail de Bontemps et al. (2005).

${ }^{22}$ Pratiquement, ils estiment une équation d'évolution du prix des MN (modèle AR(1)) dans lequel ils introduisent une variable indicative de la présence ou non de MDD. Le coefficient de cette variable leur indique l'effet prix de l'introduction (existence) d'une MDD.

${ }^{23}$ Ils étudient également l'effet de l'entrée d'une nouvelle MN. Ils obtiennent des résultats du même type puisque dans 34 cas sur 63, ils concluent que l'entrée d'une nouvelle MN s'accompagne d'une augmentation du prix des autres MN.

${ }^{24}$ Certains travaux réalisés sur un nombre très limité de produits concluent à un effet négatif (de l'entrée des MDD) sur le prix des MN. Ainsi, Chintagunta, Bonfrer et Song (2001) étudient l'impact de l'introduction des 
MDD sur le marché de certaines céréales pour le déjeuner (avoine). Ils montrent que l'introduction des MDD s'accompagne d'une baisse du prix de la marque nationale, d'une diminution des actions de promotion sur cette marque et d'un maintien des marges des distributeurs sur la marque nationale.

${ }^{25}$ Carrefour a développé également une gamme de produits de terroir pour lesquels l'enseigne a créé un nom spécifique (Reflets de France). D’autres enseignes font de même (Casino avec les produits 'Destinations saveurs' ou Leclerc avec les produits 'Nos régions ont du talent').

${ }^{26}$ A l'inverse, pour les marques de producteur, le distributeur ne choisit pas les caractéristiques du produit. Son action se ‘limite’ à la sélection des produits qu'il distribue et leur mise en valeur dans les rayons.

${ }^{28}$ Dans le cadre de ce modèle, la qualité de la marque nationale est exogène et n’est pas choisie stratégiquement.

${ }^{29}$ Dans tous les cas, le distributeur est en situation de monopole.

${ }^{33}$ On peut invoquer le caractère incomplet des contrats en général pour justifier cette impossibilité du producteur de s’engager sur un contrat vis-à-vis du distributeur.

${ }^{36}$ Selon Cotterill, Putsis et Dhar (2000) ces résultats contre-intuitifs peuvent également provenir d'un traitement économétrique inapproprié qui n’intègre pas la simultanéité de la demande et des interactions compétitives entre les acteurs du marché. Travaillant sur des données du même type, et traitant le problème de simultanéité, ces auteurs trouvent une relation positive entre la part de marché des MDD et l'écart de prix entre MN et MDD.

${ }^{39}$ Par exemple, le producteur va prendre en charge une partie des coûts comme le packaging ou encore il va aider efficacement le distributeur dans la définition précise d'un cahier des charges.

${ }^{43}$ Des travaux sur les interactions stratégiques producteurs-distributeurs rejettent l'hypothèse de prix linéaires, Vilas-Boas (2005), Bonnet et al. (2005), Bonnet et Dubois (2006). 